\title{
La comunicación y gestión de crisis. Notas comparativas entre el Caso Prestige y la tragedia del Golfo de México
}

\author{
Ana Belén Fernández Souto
}

Recibido: 19 de julio de 2012 Aprobado: 8 de septiembre de 2012

\begin{abstract}
Resumen
Proponemos plantear una reflexión sobre las técnicas e instrumentos de Relaciones Públicas utilizadas en la gestión de crisis de dos vertidos petrolíferos: el caso del Prestige y el del Golfo de México.
\end{abstract}

Separadas por ocho años de diferencia, la gestión política e institucional de la crisis en ambos casos ha sido bien distinta, y sus respectivas fases de recuperación van por derroteros muy alejados.

Partiendo de diversas investigaciones materializadas en las universidades gallegas en las que el caso de la crisis del Prestige fue analizada desde múltiples puntos de vista de la comunicación (periodismo, acciones publicitarias, acciones de relaciones públicas, repercusión institucional, efectos propagandísticos, etc.), intentaremos realizar una comparativa sobre la gestión de la situación en ambos casos.

Además, tomaremos como referencia el análisis de otras investigaciones que realizan comparaciones similares entre situaciones crisis en el sector petrolero, caso de las existentes para el caso Prestige/Exxon-Valdez.

Con esta investigación planteamos conseguir un documento en el que se exponga un breve análisis comparativo de la gestión de crisis de ambos casos, y en la que se reconozcan claramente las principales acciones, instrumentos, tácticas y técnicas de relaciones públicas aplicadas en cada uno de ellos.

Igualmente, esperamos que el estudio permita observar la evolución en la gestión de crisis en este sector tan específico y proclive a problemas medioambientales, ya que en los últimos años el desarrollo de los websites, blogs, redes sociales, etc. ha resultado claves en la comunicación de crisis.

Palabras clave: comunicación de crisis, relaciones públicas, crisis, petróleo, comunicación.

* Esta investigación comparativa proviene de una de las líneas de investigación del grupo CP2 de la Universidade de Vigo, especializados en la gestión y comunicación de/en crisis y las relaciones públicas. La financiación existente para el mismo proviene de la Convocatoria de la Cátedra Filgueira Valverde promovida por la propia Universidade de Vigo con entes sociales e institucionales como la Diputación de Pontevedra o Caixanova.

** Profesora titular de la Facultad de Ciencias Sociales y de la Comunicación de la Universidad de Vigo, España. Investigadora en las áreas de Comunicación, Relaciones Públicas, protocolo y gestión de crisis. Correo electrónico: abfsouto@uvigo.es 


\title{
Communication and crisis management. Comparative notes between Prestige Case and the tragedy of Mexican Gulf
}

\begin{abstract}
A reflection on public relations techniques and instruments used in crisis management of two petroleum spillages: Prestige and Mexican Gulf cases are proposed. These two cases occurred with a difference of eight years; the political and institutional management of the crisis has been different and its recovering phases have taken different ways. Going from different researches carried out in Galician universities in which the Prestige case was analyzed from different points of view of communication (journalism, publicity, public relations, institutional repercussion, advertising effects, etc.), this article intends to show a comparison on the management of the situation in both cases. Besides, other researches which make similar comparisons between crisis situations in petroleum sector will be taken as reference, for example the case of Prestige/Exxon-Valdez. With this research it is intended to prepare a document in which a short comparative analysis of the crisis management of both cases and in which the main actions, instruments, tactics, and techniques of public relations applied on each one of them are clearly recognized. It is also expected that this study allows observing the evolution in crisis management in this specific sector and disposed to environmental problems, since in the last years the development of websites, blogs, social networks, etc. have been keys in crisis communication.
\end{abstract}

Key words: Crisis communication, Public relations, crisis, petroleum, communication 


\section{Introducción/Introduccion}

Para el presente artículo planteamos una reflexión sobre dos casos concretos a analizar. Nos referimos al estudio de la gestión de crisis llevada a cabo desde el punto de vista de la comunicación para dos catástrofes petroleras acaecidas en Galicia, durante el 2002, y en el Golfo de México, en 2010.

A pesar de contar con casuísticas muy diferentes, ambos casos son dignos de estudio desde el ámbito de las relaciones públicas como ejemplo de la mala gestión institucional, en el primer caso, y empresarial en el segundo.

Para ello, haremos un resumen de los dos accidentes, incluyendo una contextualización geográfica, temporal, política y social para, a continuación, centrarnos en el análisis de las políticas comunicativas desarrolladas para salvar la situación adversa en cada uno de los dos casos.

Finalmente, presentaremos una serie de conclusiones que, actuando a modo de evaluación, puedan resultar una aportación al ámbito científico, pero sobre todo, a la aplicación práctica de procesos comunicativos en la gestión de crisis para eventos de esta tipología.

\section{Objetivos/Objectives}

1. Pretendemos realizar una breve comparación sobre la gestión comunicativa y de relaciones públicas aplicadas en las crisis petroleras del Prestige (Galicia, 2002) y Golfo de México (Golfo de México, 2010).

2. Materializar un documento-referente sobre los principales errores y aciertos de la gestión de crisis para ambos casos. Este documentoreferente será el culmen de la reflexión sobre la ejemplaridad de ambos casos como lo que NO se debe hacer en situaciones de gestión de crisis, desde el punto de vista de las relaciones públicas.
3. Confirmar la hipótesis de partida que indica que ambos casos resultaron paradigmas de lo que no se debe hacer en una crisis: desde el punto de vista institucional y político, para el caso del Prestige, y desde el punto de vista empresarial, para el caso del Golfo de México y BP.

\section{Metodología/Metodology}

La investigación se desarrolló en tres fases principales: recopilación de material sobre cada una de las crisis objeto de estudio (publicaciones, acciones desarrolladas, hechos en sí...); vaciado de fuentes científicas para justificar la investigación, y una última fase en la que se llevó a cabo el análisis comparativo en la gestión de ambas situaciones, método comúnmente utilizado en las ciencias sociales.

Para abordar el tema, acudimos a fuentes bibliográficas en materia de marketing y comu nicación de/en/durante/post crisis, además de indagar en otras fuentes biliográficas, hemerográficas y demás fuentes primarias (entrevistas) sobre la irrupción de las redes sociales, los blogs $y$, en general el mundo de Internet en la gestión de crisis de este alcance.

\section{El caso Prestige: Galicia 2002/ Prestige accident: Galicia 2002}

El Prestige era un barco petrolero de casco único que el 19 de noviembre de 2002 se partió en dos frente a la costa gallega, derramando casi toda su carga al mar a tan solo $250 \mathrm{~km}$ de tierra firme.

La parte afectada de la costa no solo tenía una gran importancia ecológica, sino también una notable industria pesquera. El 2 de enero de 2003, las manchas de combustible estaban a 50 kilómetros de la costa, y días más tarde, llegaron a las playas gallegas. La marea negra provocada por el vertido resultante causó una de las catástrofes medioambientales más grandes de la historia de 
la navegación, tanto por la cantidad de contaminantes liberados como por la extensión del área afectada, una zona comprendida desde el norte de Portugal hasta las Landas de Francia. Este desastre tuvo especial incidencia en Galicia, donde causó, además, una importante crisis política.

\subsection{Las primeras horas de la crisis/ The first hours of the crisis}

Durante las primeras horas del desastre, tanto el Gobierno gallego como el español se limitaron a minimizar las consecuencias. Desde el primer momento, la política comunicativa se caracterizó por la negación de los hechos y la falta de transparencia, tanto desde el punto lingüístico (por ejemplo, no se hablaba de "marea negra" sino de "manchas" o "hilillos de plastilina"), actitudinal (ausencia de las principales autoridades en la "zona cero", en algunos casos como el del Presidente de la Xunta o el Vicepresidente de Gobierno, disculpada por estar de cacería), científico y de hechos (se afirmó institucionalmente que no llegaría la marea negra a la costa gallega, se negó la existencia de catástrofe ecológica, etc.).

Mientras las instituciones públicas españolas optaban por esta vía, la empresa o empresas responsables del barco se decidieron por la estrategia del silencio.

\subsection{Los medios de comunicación tradicionales / The traditional mass comunication}

Con una noticia de este alcance, los medios de comunicación locales, nacionales e internacionales hicieron un seguimiento exhaustivo de la información.

A pesar de ello, se acusó a la prensa gallega y española, en general, de no actuar por cuenta propia en la gestión de la información y de depender, en gran medida, de los datos facilitados desde los diferentes gobiernos; tanto fue así que incluso los medios de comunicación más renombrados de Galicia, caso de La Voz de Galicia o Faro de Vigo, fueron señalados por algunos sectores ga- llegos durante los primeros días de la catástrofe, como cómplices y encubridores de las mentiras del Gobierno.

Este fue el motivo por el que la opinión pública acudió de forma masiva a consultar fuentes extranjeras, fundamentalmente prensas lusa y francesa que hacían el seguimiento de la noticia aportando datos que no se publicaban en la prensa nacional.

Con el paso del tiempo, ya en plena fase crónica de la crisis, algunos medios empezaron a ofrecer informes más independientes, a la vez que surgieron nuevas voces, sobre todo a través de la Red de Redes, que aportaban otros datos, más alejados de las premisas marcadas por los gobiernos gallego y nacional.

\subsection{Internet/ The Internet}

Para el caso del Prestige, debemos indicar que Internet no contaba con el peso comunicativo que tiene hoy día. En ese sentido, la política comunicativa e institucional de la Xunta y el Gobierno central para la Red se limitó al uso de las mismas estrategias que para el resto de medios y soportes: primero la negación de la catástrofe y, después, la minimización de los hechos.

En 2002, la Asociación de Empresas Consultoras en Relaciones Públicas y Comunicación llegó a la conclusión de que se cometieron varios errores en la gestión de la comunicación de la crisis del Prestige, entre ellos, la falta de transparencia de las administraciones o las declaraciones minimizando la magnitud de la crisis por parte de portavoces oficiales, además de la escasa o nula utilización de Internet como medio de comunicación rápido y versátil. Incluso el Colegio Oficial de Periodistas de Galicia utilizó Internet, en aquel entonces, para criticar el apagón informativo al que estaba siendo sometida la sociedad.

Aunque en esas fechas, la web 2.0 ni siquiera se encontraba en pañales, la red era un hervidero de blogs y foros donde la gente comentaba las 
noticias y el quehacer diario. En ese momento, la falta de información fiable respecto a la crisis del Prestige hace que los ciudadanos españoles acudan a Internet en busca de páginas extranjeras (francesas y portuguesas, fundamentalmente) para recopilar información veraz.

En ese sentido, cabe recordar el papel que tuvieron webs como vieiros.com, que se erigió como uno de los estandartes de la información veraz y los datos contrastados con entrevistas y comentarios de científicos de todo el país. Lo mismo ocurrió con el site de www.plataformanuncamais.org o blogs como www.prestige.blogalia. com; www.accede.org o www.adega.info.

Para contrarrestar esta pujanza, la Xunta creó dos páginas: una web institucional de carácter informativo www.ccmm-prestrige.cesga.es y otra que en principio parecía más altruista y con vocación ecologista www.fundacionarao.xunta.es (que fue disuelta a los dos años del desastre). En la primera se informaba a diario de la situación del vertido, y también de las rías gallegas. Asimismo, era el lugar por el que tenían que pasar los voluntarios para hacer oficial su "voluntariedad". Por otra parte, Fundación Arao sirvió para publicitar todo tipo de estudios, colaboraciones científicas avaladas por la Xunta, o proyectos de colaboración con los ayuntamientos afectados. En ambas webs, los mensajes eran siempre positivos, aceptando el desastre, pero no la culpa.

La solidaridad de todo el país se organizó a través de Internet, ya que mientras el Gobierno negaba la necesidad de voluntarios desde la propia www.ccmm-prestige.cesga.es, otras webs pedían más medios y más manos.

\subsection{La respuesta social / The social response}

La respuesta social ante la crisis medioambiental y la crisis política no se hizo esperar, y pronto la marea de voluntarios empezó a llegar a Galicia para limpiar sus playas y costas. Tal y como adelantábamos, el voluntariado se organizó básicamente a través de Internet, si bien cabe reconocer que al amparo del Gobierno autonómico.

Aparte de esta respuesta voluntaria, surgió el colectivo Nunca Máis, que contó con una gran actividad movilizadora para protestas y manifestaciones contra el Gobierno autonómico y que incluso llegó a presentar acciones legales contra los gestores de la crisis.

\section{El caso Golfo de México: 2010/ The disaster of the Gulf of Mexico}

\subsection{Las primeras horas/ The first hours}

El 20 de abril de 2010 once trabajadores de BP desparecen en la explosión de una plataforma petrolífera en el Golfo de México, dos días después, la plataforma de hunde; en ese momento, 1.000 barriles de crudo salen cada día a la superficie. Diez días después del inicio de la crisis, la marea negra llega a la costa.

La empresa BP asume su culpabilidad y afirma que asumirá también todos los gastos derivados del posible daño que pueda causar la crisis.

La crisis se cierne en torno a la empresa petrolífera y las presiones institucionales se centran en ella como última responsable.

El 2 de mayo el Presidente Obama visita la costa para reunirse con los principales afectados; esta visita volvería a repetirse el 28 de mayo, cuando ya la mancha de petróleo llega al delta del Misissipi y aparecen los primeros indicios de marea negra en la costa de Florida.

A estas alturas, BP empieza a reconocer sus errores y afirma que no contaba con equipos suficientes para hacer frente a la situación. Ya el 17 de junio, comparece por primera, vez ante una comisión del Congreso, el Consejero Delegado de BP, Tony Hayward, que se limita a pedir disculpas. 
Al día siguiente, el Presidente de BP le relega para tomar él mismo las riendas.

Un mes después, el 16 de julio BP anuncia que ha conseguido sellar la fuga de crudo por completo, aunque el paso del tiempo lo desmiente.

El 19 de septiembre, casi cinco meses después, el gobierno de EE. UU. anuncia que el pozo de petróleo causante de la marea negra ha sido cerrado definitivamente.

\subsection{El papel de Internet durante la crisis/ the rol of the Internet during the crisis}

En el caso de la crisis del Golfo de México podemos diferenciar claramente las políticas gestoras y comunicativas llevadas a cabo desde el Gobierno y desde la empresa BP.

Por su parte, el Gobierno norteamericano hizo desde un primer momento lo más acertado: informar desde el primer momento, no falsear información, implicarse en el seguimiento y limpieza de la marea negra, exigir responsabilidades a la empresa, evaluar la crisis constantemente y mantenerla en perspectiva, así como crear espacios públicos para que todos los interesados pudiesen disponer de la última noticia, caso de la web www. restorethegulf.gov.

BP, por el contrario, nunca controló el flujo de la información y no supo aprovechar el tirón de las redes sociales. Muestra de ello es la siguiente frase "la seguridad es nuestra principal preocupación. Bueno, nuestras ganancias y luego la seguridad. Oh no, nuestras ganancias, nuestra imagen, y después la seguridad. Como sea... nos importa". Esta "tweet" se publicó en una cuenta falsa a nombre de BP en la que el tradicional logo verde de la compañía aparecía en negro y con un derrame. Casi un año después sigue activa y a pleno rendimiento: cuenta con 186.449 seguidores (datos de enero 2010) mientras que la cuenta oficial de la petrolera británica cuenta con unos 18.000 .
A pesar de todo, BP lo intentó y abrió perfiles en las principales redes sociales, flickr, facebook, youtube y twitter.

\subsection{La gestión de la crisis/ The crisis management}

La gestión de BP cayó en todos los errores posibles: faltó transparencia informativa; la reacción se hizo esperar demasiado; mostraron demasiada arrogancia, lo que implicó la pérdida de imagen positiva de la empresa; falló el portavoz, tanto en su gestión comunicativa con los medios como con las instituciones públicas; se utilizaron "falsos expertos", lo que puso aún más en entredicho a la empresa; compraron publicidad en lugar de ofrecer información; BP puso anuncios en Google relacionados con búsquedas de términos como "vertido de petróleo", "voluntario" o "reivindicaciones", además de gastar más de 50 millones de dólares en anuncios para televisión, etc. La imagen de la organización todavía está en entredicho.

Frente a ello, la gestión institucional del Gobierno fue totalmente inversa, apostando siempre por la transparencia, la implicación de las autoridades y la verdad.

\section{Comparativa en ambos casos y planteamiento del documento de reflexión/Comparative of both cases and the approach to the discussion paper}

Como vemos, ambos casos son muy diferentes; sin embargo, cuentan con varias similitudes que permiten estudiarlos de forma comparativa: los dos son importantísimos desastres naturales y medioambientales, cuyas consecuencias todavía están por verse; en ambos se implicaron directamente las empresas (armadora/barco/compañía petrolera) y los gobiernos institucionales (autonómico/regional/nacional); sin embargo, la implicación y, sobre todo, la gestión comunicativa de la crisis han conseguido que la sociedad apunte 
en un caso como culpable a la administración autonómica y en el otro, a la propia petrolera. ¿Cuáles han sido, pues, los motivos que han llevado a esta pérdida de imagen para unos y no para otros?

\subsection{Decir la verdad y establecer una posición clara/ Tell the truth and stablish a clear position}

En una crisis como la del Prestige no se puede negar lo evidente y afirmar tajantemente que la crisis no era tal. Los primeros en minimizar hasta el ridículo la realidad fueron los propios líderes políticos (Conselleiros de la Xunta de Galicia, Delegado del Gobierno, Vicepresidente del Gobierno, Ministros, etc.). En esta primera fase, no se asumieron responsabilidades y se intentó culpar al capitán del barco.

Poco a poco, la información y los datos fueron haciéndose públicos, lo que evidenció la falta de rigor y la falta a la verdad, y puso en entredicho a portavoces y a instituciones públicas como la Xunta de Galicia o la Vicepresidencia del Gobierno. Tanto fue así que ya en enero de 2003 el barómetro del CIS indicaba que una de cada cuatro personas encuestadas tenía la sensación de que los principales responsables de la catástrofe fueron el capitán, la armadora y la empresa fletadora; otra creía que el Gobierno español debía asumir toda la responsabilidad; la tercera persona creía que la culpabilidad la tuvieron las actuales leyes de navegación marítima, en tanto que la última persona no supo a quién adjudicar la responsabilidad. El caso es que la responsabilidad quedó dirimida entre las distintas instituciones públicas, a la espera de que la justicia dicte un veredicto final.

Tal y como adelantábamos, la actuación del Gobierno norteamericano fue todo lo contrario, apostando por favorecer la verdad y no ocultar información. Sin embargo, la petrolera BP no optó por esa misma estrategia comunicativa, intentando desde un primer momento el minimizar los hechos y ocultar su escasez de recursos para hacer frente a la situación; tanto fue así que incluso llegó a publicar fotografías falsas (caso de las fotos del Centro de control de crisis para demostrar que lo tenían todo vigilado y controlado, fotografía retocadas y falseadas a través de programas de retoque fotográfico) que rápidamente fueron distribuidas a través de blogs y redes sociales y que dejaron en evidencia la falta de coherencia por parte de la empresa.

\subsection{Pedir ayuda/ Ask for help}

En el caso del Prestige, la Xunta se negó a pedir ayuda desde el primer momento, tanto para la gestión comunicativa como para la propia gestión física de la catástrofe (necesidad de barreras de contención, voluntariado, material técnico, expertos y profesionales, etc.). Así pues, todos los buques especializados vinieron de países extranjeros; la marea de voluntarios llegó de toda España y parte del extranjero, igual que ocurrió con el ejército, dándose el caso de que llegaron antes los efectivos belgas que nuestras propias tropas.

Parecía increíble que con la experiencia de la costa gallega en el siniestro de buques petroleros no contase con un plan de crisis claro, efectivo y suficientemente contrastado.

Por el contrario, BP contaba con un plan de crisis, sin embargo resultó estar poco ajustado a este caso concreto, ya que calculaba que el peor vertido posible del pozo sería de 162.000 barriles al día, casi el triple del que realmente se produjo y la compañía aseguraba que estaría en condiciones de recuperar casi 500.000 barriles al día, utilizando la tecnología estándar del sector, por lo que aunque se produjera el peor vertido posible, no supondría una amenaza grave para los caladeros ni para la fauna del Golfo, entre la que citaba morsas, nutras marinas y leones marinos que, evidentemente no hay en el golfo de México. El plan de BP mencionaba, además, como asesor 
en caso de emergencia a un biólogo marino que había muerto hacía años y ofrecía la dirección de una página de Internet japonesa de entretenimiento como fuente de equipamiento en caso de un vertido accidental. Evidentemente, cuando se produjo el vertido, la capacidad de reacción de BP fue muy inferior a la anunciada y su gestión de la crisis resultó nefasta.

Durante una crisis de este calado, el contar con informes, técnicos y, en general, apoyos externos a la organización aporta claramente un valor añadido ligado al compromiso social y al compromiso con la verdad.

\subsection{Estar presente en el lugar de los hechos / Be in the place of facts}

Los grandes ausentes de la crisis del Prestige fueron los responsables políticos: Manuel Fraga como Presidente de la Xunta no se dejó caer por las costas gallegas de "chapapote", y el Presidente del Gobierno, José María Aznar se presentó 30 días después, con una visita "obligada" de menos de 4 horas, en la que ni siquiera pisó una playa afectada, ya que la famosa visita se limitó a un paseo en avión desde el que observar la magnitud del desastre.

En el caso del Golfo de México, el propio presidente Obama visitó a los afectados en dos ocasiones e, incluso, la primera Dama realizó algún viaje de apoyo a los damnificados. Sin embargo, los directivos de la BP no se dejaron ver demasiado, agravando además este hecho por la mala elección del portavoz, que carecía de carisma y, sobre todo, de empatía y que, al poco tiempo de estallar la crisis, tuvo que ser relegado de su cargo.

En este tipo de crisis con consecuencias sociales, económicas y medioambientales tan importantes, los líderes deben implicarse casi de forma personal, consiguiendo que los afectados perciban su preocupación y demostrado su empatía.

\subsection{Centralizar las comunicaciones/ Centralize communications}

Durante el Prestige la figura del portavoz resultó muy interesante, no por su función, sino por la gran variedad de personas que ostentaron este cargo. En ese sentido, se documentan intervenciones del Conselleiro de Pesca, Fernández de Mesa (Delegado del Gobierno en Galicia), Álvarez Castos (Ministro de Fomento), Jaume Matas (Ministro de Medio Ambiente) o Mariano Rajoy (Vicepresidente del Gobierno). La ausencia de una cabeza visible que sea la voz de la institución resulta un fallo importantísimo, ya que denota una falta de organización y previsión, además de redundar en una imagen negativa de la propia institución.

En el caso de BP, el portavoz Tony Hayward no supo transmitir empatía y tuvo que ser relegado en plena fase aguda de la crisis. A pesar de todo, desde la BP se realizaron intentos de centralización de comunicaciones hacia los medios de comunicación en las oficinas del centro de operaciones de British Petroleum en Horma, Luisiana, aunque con una escasa previsión y reacción, lo que derivó en que fuesen los propios medios de comunicación los que llevasen la iniciativa comunicativa y no la empresa. De esta forma, el papel informativo de la BP prácticamente quedó relegado a un segundo plano en que se limitaban a desmentir o confirmar los datos que aportaba la prensa.

\subsection{Cooperar con los medios/Cooperate with mass media}

Tal y como afirmamos anteriormente, en el seguimiento informativo de la contaminación del Prestige la información más veraz se conseguía en fuentes portuguesas y, especialmente, francesas, dado que los medios de comunicación nacionales limitaban mucho su información a la facilitada desde las instituciones públicas. Este hecho fue cambiando a medida que la crisis avanzaba con el surgimiento de nuevos diarios, blogs, webs y demás voces discrepantes... 
A este hecho debemos añadir el que durante los primeros días de la crisis del Prestige, se vetó incluso la presencia de las televisiones en la costa, lo cual pone de manifiesto que la transparencia comunicativa y la atención a la verdad quedaban relegadas a un segundo plano, siempre por detrás de intereses políticos.

Similar situación se dio en la crisis del Golfo de México cuando se prohibía a los fotógrafos acercarse a menos de 20 metros de las barreras flotantes, situación denunciada en foros y blogs. A pesar de todo, en el caso de BP la reacción fue rápida (al contrario que con el Prestige, pero no fiel a la realidad).

\subsection{No perder la perspectiva de la fase de recuperación de la crisis/ Remember the fase of the recuperation of the crisis}

Muchas veces ocurre que las organizaciones, ya sean de carácter público o privado, pierden de perspectiva la crisis, cuando todavía está en la fase de recuperación o dando sus últimos coletazos.

En este sentido, para el caso del Prestige las autoridades no deben perder de vista las posibles consecuencias medioambientales que pueda tener el vertido en las costas gallegas, implicando en este sentido al sector pesquero (conserveras, pesca de bajura), al turismo, etc.

Igualmente, en los últimos años se vienen haciendo públicos diversos informes sobre las posibles repercusiones en salubridad que pueda tener la crisis del Prestige. En este sentido, destacamos un informe de la Sociedade Galega de Homeopática que prevé la aparición de cánceres de piel y pulmón relacionados con el contacto con el fuel del Prestige. De este estudio se desprende que hay miles de personas que pueden verse afectadas a medio o corto plazo, tanto voluntarios, como personas que viven en la costa. La toxicóloga Amparo Casal denuncia la actitud de las autoridades con respecto a la catástrofe y la falta de protección para la salud de los voluntarios y habitantes de la zona.

Tampoco se puede descuidar el proceso ju dicial, que sigue abierto. La investigación judicial sobre cómo se gestionó la crisis del Presgite y las posibles responsabilidades gubernativas se lleva a cabo en el Juzgado de Instrucción $n^{\circ} 1$ de Corcubión (La Coruña), tras admitir a trámite la querella presentada por la Plataforma ciudadana "Nunca Máis" contra el delegado del Gobierno en Galicia, Arsenio Fernández de Mesa, el director general de la Marina Mercante, José L. López Sors y el capitán marítimo de La Coruña cuando sucedieron los hechos, Ángel Del Real. Esta querella también está dirigida contra el capitán del buque, sus propietarios, el armador y el fletador, pero considera a los tres responsables del Gobierno culpables de agravar las consecuencias de la catástrofe al haber enviado el petrolero lejos de las aguas territoriales españolas.

Además, la federación de partidos de Izquierda Unida ha presentado denuncia en el mismo juzgado contra los Ministros de Fomento y de Medio Ambiente por "delitos ecológico, prevaricación ecológica, daños a la fauna silvestre, daños a un espacio natural protegido y riestos", todos ellos tipificados en el Código Penal.

Ante estos hechos, resulta evidente que la crisis del Prestige está tocando fin, pero todavía está en fase de recuperación y sería un grave error por parte de las administraciones públicas y las empresas (barco, propietarios, armadora, etc.) implicadas el no prestarle atención a estos últimos coletazos y no el mantener una política comunicativa adecuada.

Respecto al caso de BP para la crisis del Golfo, su recuperación todavía está en una fase muy inicial, dado que hace menos de un año que explotó en su fase más cruda y aguda. Tendremos que esperar varios años para ver cuáles serán los derroteros que la petrolera británica tendrá que 
atender para salir lo más airosa posible de esta situación.

\section{Conclusiones/Conclusions}

Existen organizaciones, tanto de carácter público como privado, que necesitan cuidar especialmente su gestión de crisis (antes, durante y después): es el caso de las empresas petroleras.

Parece que la clave para salir airosos de una situación adversa en el menor tiempo posible es estar preparados con antelación y previsión, mediante la gestión de planes de crisis, y en los últimos tiempos, atendiendo a la web, debido a su gran capacidad para transmitir información y movilizar a la opinión pública.

Esta planificación es fundamental y su ausencia quedó demostrada en los dos casos concretos analizados en este artículo: la crisis del Prestige (Galicia 2002) y la Crisis del golfo de México (Golfo de México, 2010).

Por los datos arrojados de la investigación, el primer ejemplo se cebó especialmente en la clase política gobernante en Galicia y, por extensión, en España en aquel entonces, como un ejemplo de mala gestión comunicativa durante una crisis, desde el punto de vista de las instituciones públicas. Por el contrario, en el derrame del Golfo, la crisis fue meramente de carácter empresarial, ya que las administraciones públicas norteamericanas apostaron desde un momento por la gestión informativa veraz y mostraron gran empatía con los afectados a través de diversos viajes institucionales, puesto que si bien en un primer momento se acusó a Obama de no comparecer a tiempo, días más tarde incluso la primera Dama, Michelle Obama, se implicó en el seguimiento del desastre. Este hecho hizo recaer toda la responsabilidad de la crisis en la empresa petrolera, la BP, cuya gestión se caracterizó claramente por la lentitud a la hora de reaccionar debido a la falta de previsión, falta de transparencia informativa, falta de coordinación, mala elección del portavoz o la falta de empatía.

En ambos casos, es importante aprender de los errores y que tanto instituciones públicas como privadas conozcan la importancia de la prevención y la preparación de planes de crisis que permitan ganar tiempo, actuar con seguridad y minimizar riesgos en el más amplio de los sentidos.

\section{Referencias documentales/ Documental references}

Capriotti, Paul (2005). "La gestión comunicativa de situaciones de crisis" en Comunicación Organizacional: Teorías y Estudios. Málaga: Editorial Clave. Antonio Castillo (coord.).

Contreras, D. (2006). "La gestión de crisis en la comunicación organizacional: el caso de Chile". Anàlisi, 34: 305-313.

Fita, Jaume (1999). Comunicación en programas de crisis. Barcelona: Gestión 2000.

Fita, Jaume (2004). "Comunicación de crisis", en LOSADA DÍAZ, José Carlos (coordinador). Gestión de la comunicación en las organizaciones. Barcelona: Ariel Comunicación-

González Herrero, Alfonso (1998). Marketing preventivo: la comunicación de crisis en la empresa. Barcelona: Bosch

Losada, J. C. (2010) Comunicación en la gestión de crisis. Barcelona: UOC

Mayntz R. Holm (1980) Introducción a los métodos de la sociología empírica. Madrid: Alianza Editorial.

Middleberg, D. (2001) Relaciones Públicas en un mundo interconectado. Estrategias de comunicación para triunfar en el espacio digital. Bilbao: Deusto.

Mitroff, I. (2005). Why some companies emerge stronger and better from a crisis: 7 essential lessons for surviving disaster. New York: Amacom

Mitroff, Ian y Pearson, Christine (1997). Cómo gestionar una crisis. Barcelona: Gestión 2000. 
Noya, J. y Prado, R. (2011). Cuál es la evaluación de la reputación de España en el mundo entre 2009 y 2011 . http:// www.realinstitutoelcano.org/wps/portal/rielcano/ contenido?WCM_GLOBAL_CONTEXT=/elcano/ elcano_es/zonas_es/ari158-2011

Palenchar, M. J. y Heath, R. L. (2007). "Strategic risk communication: adding value to society" in Public Relations Review, 33 (2): 120-129.

Taylor, M. y Kent, M. (2007). "Taxonomy of mediated crisis responses", in Public Relations Review, 33: 140- 146.

Tuñez, Miguel (coordinador) 2007. Comunicación preventiva. Planificación y ejecución de estrategias de información interna y externa ante situaciones de crisis. A Coruña: Netbiblo

Ulmer, R.; Seeger, M. y Sellnow, T. (2007). "Post-crisis communication and renewal: expanding the parameters of post-crisis discourse" in Public Relations Review, 33: 130 134.

Villafañe, J. (2004). La buena reputación. Claves del valor intangible de las empresas. Madrid: Pirámide.

Xifra, J (2005) "La gestión de conflictos potenciales como estrategia de comunicación corporativa: el caso Microsoft" in ZER, No 18.

Websites visitados entre octubre y diciembre de 2010:

www.webs.uvigo.es/h06/tsfp/es-en/efectos_es.html

www.nationalgeographic.com.es

www.elmundo.es/especiales/2002/1 1/ecología/prestige

www.accede.org/prestige/prestige.htm

www.fundacionarao.xunta.es

www.ccmm-prestige.cesga.es www.prestige.blogalia.com

www.adega.info

www.plataformanuncamais.org

www.vieiros.org

www.greenpeace.org

www.sicprestige.iim.csic.es

www.terra.es/personal/darsoft/chapapote/fotos.html

www.le-cedre.fr/fr.prestige/z_photo.htm

www.esa.int/export/esaCP/ESAGFD7708D :index 0. html

www.aurensa.es/archivo/2002-aa-17 CostaDaMorte/2002-11-17 CostaDaMorte.htm

www.le-cedre.fr/fr./prestige/fichas.htm

www.elmundo.es/america/2010/06/12/estados unidos/1276374222.html

www.comocomunicar.com

http://gestion.pe/noticia/492204/bp-intenta-aprovehcar-derrame-internet

http://www.netambulo.com/2010/07/22/la-crisis-delvertido-de-petroleo-de-bp-y-sus-fotos-trucadas-conphotoshop

www.restorethegulf.gov

www.bp.com/extendedsectiongenericarticle.do?categ oryld $=40$ Econtentld $=7061813$

www.rrppnet.com.ar/crisisgolfodemexico.htm 
